\title{
CUIDADOS DE ENFERMAGEM A PACIENTES EM PRÉ-OPERATÓRIO: PROPOSTA DE CHECKLIST
}

\section{NURSING CARE PATIENTS IN PRE-OPERATIVE: MOTION FOR A CHECKLIST}

\author{
Neiva Junkes Hoepers, Hercília Machado Baccin, Valdemira Santina Dagostin, Paula \\ lopi Zugnio, Maria Salete Salvaro
}

\section{RESUMO}

A segurança para o paciente no pré-operatório é um dos itens de maior relevância e imprescindível para a vida das pessoas que precisam submeter a uma cirurgia, tanto no período mediato como imediato da cirurgia. Para isso é importante à existência de um instrumento que normatize todos os cuidados neste período, podemos chamar de 'cheklist' ou protocolo do cuidado pré-operatório, que possa ser utilizado tanto pela equipe de enfermagem, como a equipe multiprofissional. A pesquisa teve como objetivo, caracterizar os cuidados de enfermagem prestados a pacientes em período pré-operatório mediato e imediato, e propor um 'checklist' para uma Clínica Cirúrgica, como forma de garantir a segurança do paciente antes do procedimento cirúrgico. Pesquisa qualitativa, descritiva, exploratória e de campo com 42 profissionais da equipe de enfermagem em uma instituição hospitalar do sul de Santa Catarina, nos meses de abril e maio de 2016. Os resultados apontaram que o serviço não possui instrumento de 'cheklist' ou protocolo para pré-operatório mediato e imediato e que houve um déficit de orientação quanto ao cuidado realizado. Foi elaborado uma proposta de 'cheklist' para ser entregue a instituição da pesquisa. Como também salientamos a necessidade da realização de educação permanente para estes cuidados com toda equipe de enfermagem.

Palavras-chaves: Cuidado pré-operatório; Enfermagem; Cirurgia Segura.

\section{ABSTRATC}

The safety for the patient preoperatively is one of the most relevant items and essential to the lives of people who need to undergo surgery, both in the middle period as the surgery immediately. This is important to the existence of an instrument that will regulate all care in this period, we can call 'cheklist' or preoperative care protocol, which can be used both by the nursing staff, as the multidisciplinary team. The study aimed to characterize the nursing care provided to patients in mediate and immediate preoperative period, and propose a checklist for a Surgery, in order to ensure the safety of the patient before surgery. qualitative, descriptive, exploratory and field with 42 professionals of the nursing staff in a hospital in the south of Santa Catarina, in the months of April and May 2016. The results showed that the service does not have instrument 'cheklist' or protocol for preoperative mediate and immediate and that there was a policy on the deficit performed carefully. a proposal for 'cheklist' to be delivered 
to the institution of the research was prepared. But also stress the need to carry out permanent education for these care in all nursing staff.

Keywords: pre-operative care; Nursing; Safe Surgery.

\section{INTRODUÇÃO}

Os avanços tecnológicos e científicos na área da saúde têm propiciado aumento significativo no número de intervenções cirúrgicas ao redor do mundo, as quais são, muitas vezes, realizadas em condições inseguras interferindo na promoção e na recuperação da saúde dos pacientes. A qualidade do cuidado e a segurança dos clientes assumem, portanto, papel de relevância, sendo reconhecida como a Era da Segurança. ${ }^{(1)}$

A preocupação com a segurança do paciente nas Clinicas e centro cirúrgico tem sido crescente, devido à elevada frequência de erros e eventos adversos que, na maioria dos casos, poderiam ter sido prevenidos. ${ }^{(2,3)}$

Em outubro de 2004, a Organização Mundial de Saúde lançou formalmente a Aliança Mundial para a Segurança do Paciente, com o objetivo de despertar a consciência e o comprometimento político, a fim de melhorar a segurança na assistência. Diante disso, os países vêm se articulando para cumprir as ações previstas nessa aliança. ${ }^{(4)}$

A Organização Mundial da Saúde e a Universidade de Harvard criaram, em 2007, o programa 'Cirurgias Seguras Salvam Vidas', considerando três elementos principais: a divulgação do conjunto de instrumentos que envolvem temas relacionados ao ambiente, materiais e equipamentos; o desenvolvimento dos profissionais de forma individual e em equipe; e estimular a realização de campanhas em ambiente local e nacional sobre o tema, considerando que os Estados membros da Organização Mundial de Saúde estejam comprometidos em programar medidas para alcançar a segurança cirúrgica. ${ }^{(2)}$

Segundo o manual para cirurgia segura da OMS (2009), o objetivo central do programa de 'Cirurgias Seguras Salvam Vidas' é definir um conjunto de estatísticas demográficas para a cirurgia que incorpore medidas de estudo e resultado, que rastreie os esforços do processo, tais como o uso de uma lista de verificação de segurança e a realização de protocolos padronizados para a assistência. ${ }^{(3)}$ 
Nos protocolos para a assistência tem-se exigido lista de verificação de segurança para o paciente, onde, tal lista, pode ser utilizado um instrumento de 'checklist' com intuito de verificação da segurança cirúrgica, podendo ser utilizado nos diversos tipos de cirurgia, independente do seu grau de complexidade, desta forma auxiliando as equipes cirúrgicas a seguirem de forma sistemática passos críticos de segurança. Como também, melhorar a assistência cirúrgica e instituir padrões de segurança que possam ser aplicados em toda instituição e para todos os procedimentos. Vindo ao encontro com o que a Organização Mundial da Saúde preconiza quanto a segurança cirúrgica. (2)

A segurança é considerada um indicador de qualidade da assistência e um dos pontos avaliados em processos de acreditação sendo obtida através da realização de medidas consideradas simples, como a checagem de materiais, de equipamentos, identificação e informação sobre o paciente. Ainda, a segurança do paciente pode ser alcançada através de "três ações complementares: evitar a ocorrência de eventos adversos; facilitar sua visualização; e minimizar os efeitos através de medidas eficazes". (2)

Quando o paciente necessita de uma cirurgia, e esta é agendada, diz- se que ele se encontra no período perioperatório, que compreende as fases préoperatória mediata, transoperatória, recuperação anestésica e pós-operatória. A cirurgia deve ser respeitada e planejada antes do procedimento para que os tempos cirúrgicos sejam respeitados e consequentemente, proporcionando segurança para 0 paciente. ${ }^{(5)}$

Destacamos aqui o primordial papel que o enfermeiro tem nessa fase préoperatória, onde a consulta de enfermagem poderá contemplar os principais pontos que teriam impacto direto na prática assistencial de enfermagem, capazes de serem implementados em diversos tipos de cirurgia e ambientes de cuidados.

Os cuidados de enfermagem que precisamos ter atenção maior, são os realizados durante o período pré-operatório mediato e imediato, que compreende desde o momento em que se decide que a cirurgia seja ela eletiva, de urgência ou de emergência, será realizada, até o momento que precede o ato cirúrgico, quando o paciente é encaminhado ao centro cirúrgico. ${ }^{(6)}$

A intervenção cirúrgica caracteriza-se como um procedimento invasivo e muitas vezes traumático para as pessoas exigindo preparo e cuidados precoces, 
desde o período pré-operatório. Desta forma, a equipe de enfermagem como toda equipe de saúde tem um papel muito importante em garantir a segurança deste sujeito, para tornar o procedimento cirúrgico mais seguro diminuindo a possibilidade de ocorrência de danos ao paciente, promovendo a realização do procedimento certo, no local e paciente corretos. Tornando necessário a utilização de uma ou de várias listas de verificação (checklist) no serviço. ${ }^{(7)}$

Todos os períodos operatórios são importantes, todavia este estudo está direcionado à fase pré-operatória, ponderando que nesta fase há uma sequência de procedimentos, preocupações e dúvidas para o paciente, que se torna propensa a um desequilíbrio físico emocional. (8)

A pessoa ao ser internada para uma cirurgia tem consigo receios e dúvidas ao saber que será submetido a um procedimento invasivo, que representa uma situação que pode ser crítica ou não, além de uma indefinição quanto aos fatos que poderão advir. Os cuidados desenvolvidos no período perioperatório variam de acordo com a especificidade cirúrgica de cada paciente, na qual o enfermeiro deve atentar para as necessidades manifestadas em cada fase deste processo, respeitando a singularidade e subjetividade do indivíduo. ${ }^{(9)}$

Nessa expectativa o papel do enfermeiro no "espaço social e técnico da unidade de clínica cirúrgica torna-se mais complexo a cada dia, à medida que necessita interligar os aspectos humanos explicitados no atendimento ao paciente, enquanto indivíduo único em suas particularidades". (9)

Assim, faz-se necessário a enfermagem enquanto ciência e profissão que cuida de pessoas, nesta fase embasarem-se em um protocolo, preciso objetivo e completo para poder prestar um cuidado com qualidade aos pacientes pré-operatório.

Diante destas colocações, de alguma forma, é preciso planejar o cuidado do profissional ou da equipe de saúde, utilizando conhecimentos teórico-práticos para qualificar a assistência e o acolhimento como estratégia de minimizar o sofrimento da pessoa que se submete a um procedimento cirúrgico.

A orientação pré-operatória eficaz ajuda o paciente a lidar com a cirurgia, desta forma reduzindo a permanência hospitalar, como também, pode aumentar a satisfação e qualidade do serviço prestado, diminuir complicações cirúrgicas, sem contar com a satisfação e qualidade de vida deste paciente. ${ }^{(6)}$ 
Com a elaboração de protocolos, pode garantir a "cirurgia segura", a qual tem uma relevância muito importante, pois, reduz à ocorrência de incidentes e eventos adversos, como também, a mortalidade cirúrgica., possibilitando o aumento da segurança na realização de procedimentos cirúrgicos, local correto, paciente correto, entre outros, por meio de um checklist com adequações, no que orienta a verificação de cirurgia segura desenvolvida pela Organização Mundial da Saúde e Ministério da Saúde. (4)

Diante destas reflexões tem-se como problema de pesquisa: Como podemos caracterizar os cuidados de enfermagem prestados a pacientes em período pré-operatório mediato e imediato de cirurgias eletivas e propor um "Checklist" para as Clinicas Cirúrgicas, como forma de garantir a segurança do paciente antes do procedimento cirúrgico.

E como objetivo, caracterizar os cuidados de enfermagem prestados a pacientes em período pré-operatório mediato e imediato e propor um checklist, a clínica cirúrgica, para procedimentos cirúrgicos como forma de garantir a segurança do paciente.

\section{METODOLÓGIA}

Pesquisa de abordagem qualitativa, descritiva, exploratória e de campo. Foi desenvolvido em uma clínica cirúrgica de uma instituição hospitalar de grande porte, na região sul de Santa Catarina. Amostra de 42 profissionais da equipe de enfermagem que realizam cuidados pré-operatórios mediatos e imediatos nesta instituição e que aceitaram participar da pesquisa assinando o TCLE e responderam um questionário com questões abertas e fechadas. Foi realizado nos meses de abril e maio de 2016, após aprovação pelo comitê de ética e pesquisa sob CAEE: 52896216.1.0000.5364. A análise e interpretação dos dados foram realizadas através da ordenação, classificação, análise e categorização dos dados e com ajuda do Windows e Microsoft Excel. 


\section{RESULTADOS E DISCUSSÃO}

Foram entrevistados 42 colaboradores com idade entre 19 e 37 anos, sendo que a maioria se encontra na faixa etária de $25 \mathrm{a}<37$ anos (90\%). Destes, 35 (83\%) eram do sexo feminino e 7 (17\%) do sexo masculino, e o estado civil 29 (69\%) casados, a formação encontrada foi 7 enfermeiros (17\%) e 35 técnicos de enfermagem (83\%).

Em relação ao tempo que o colaborador atua na instituição, 33 (78\%) estão mais de dois anos na instituição, 4 (10\%) deles de 01 a 02 anos e somente 01(01\%) de 6 meses a 01 ano, enquanto que os outros 4 (10\%) de 3 meses a 6 meses.

E quanto ao tempo de atuação no setor cirúrgico, 28 (67\%) 2 anos ou mais, 5 (12\%) de 01 ano a 02 anos, $03(07 \%)$ com 6 meses a 01 ano e os outros $6(14 \%)$ de 3 meses a 6 meses.

Verificou-se que os trabalhadores do estudo, confirmou a história do trabalho da enfermagem caracterizado sua maioria por mulheres, como também, apresentado no estudo de Maciel. ${ }^{(10)} \mathrm{E}$ por apresentar um número significativo de casados e mulheres, podemos nos remeter que estes trabalhadores podem estar desenvolvendo dupla jornada de trabalho, desta forma levando a uma sobrecarga de trabalho.

No que diz a idade, podemos ver que essa amostra mostrou profissionais jovens, demostrando que a busca pelo trabalho em idade jovem, nos remete em formação de uma família e vindo ao encontro no que é colocado por outro estudo, que os profissionais buscam trabalhar antes de atingir a terceira idade acreditando que com o tempo perdem sua vitalidade e indisposição para projetos de vida.(11)

Quanto as respostas da equipe de enfermagem, quanto a assistência de enfermagem em pré-operatório de cirurgias em seu período mediato e imediato, elaborou-se as categorização dos dados com os seguintes temas: Assistência de enfermagem ao paciente no período pré-operatório; $O$ papel da equipe de enfermagem frente ao cuidado no período pré-operatório; Rotina ou checklist do serviço quanto o cuidado no período pré-operatório; Acompanhamento e orientação à família do paciente; Outros cuidados realizados pela equipe de enfermagem. 
Para preservar a identidade os pesquisados, colocamos a letra "E" (Enfermagem) seguido de um número (referindo-se ao número dos sujeitos da amostra $=41$ ), para podermos descrever os pesquisados.

\section{Tema I - Assistência de enfermagem ao paciente no período pré- operatório}

Quando perguntado sobre a visão da equipe de enfermagem em relação à assistência de enfermagem, predominou o preparo técnico pré-operatório dos pacientes antes de entrar no centro cirúrgico, respondido por 16 deles. Seguido pela importância de se ter um protocolo ou checklist (13 profissionais), vindo depois a segurança psicológica e a emocional (12 profissionais), sendo que um deles não respondeu.

Destacamos algumas das falas dos pesquisados: E6 - "Apresentação ao paciente, explicar ao paciente como vai ser, perguntar para ele o que ele vai fazer (tipo de cirurgia) local onde vai operar, peso, jejum, alergias, passar tranquilidade." E38 - "Deveria ser mais explicado e orientado, através de exemplos vivenciados, muitas vezes o paciente é encaminhado para o Centro Cirurgico e ele não sabe o que será realizado."

Sobre a importância de se ter um protocolo ou checklist, para o cuidado com paciente no preparo para procedimento cirúrgico respondido por 13 profissionais, como a tricotomia, banho com clorexedine, se faz uso alguma medicação, se tem alergia, saber o seu peso e altura, se hipertenso, diabético, se fez avaliação anestésica, se assinou termo de consentimento, colocamos algumas de suas falas. $\mathrm{E}$ 41 - "Realizar o checklist, perguntar se o paciente é alérgico a medicações se usa prótese dentária se está em jejum de 8 a 12 horas. Confirmar cirurgia a ser realizado com paciente." E15; E14; E39 - "Todo preparo pré-operatório, como banho com clorexedine, tricotomia os que forem necessários, retirada de prótese, orientar sobre o jejum de 8 horas, orientações gerais quanto à cirurgia e guardar os pertences e dar apoio emocional". E12, a segurança psicológica, a parte emocional, a segurança que devemos passar para o paciente quando está sendo cuidado pela equipe multiprofissional, em todos os momentos quando chega ao hospital à porta de entrada será sua primeira impressão do cuidado com sua pessoa. E14 . "Conforto do paciente, 
orientação sobre o procedimento a ser realizado. Prepará-lo para o procedimento específico da cirurgia como tricotomia, atenção aos sinais vitais."

Devemos considerar a importância de se fazer um pré-operatório adequado, para segurança do paciente. Pois, a internação hospitalar é considerada pelo indivíduo que a vivencia, como sendo experiência desagradável, pois, é permeada por vários fatores: medo do desconhecido, utilização de recursos tecnológicos invasivos e dolorosos; linguagem técnica que aumenta a ansiedade da pessoa em relação a sua patologia, desconforto de estar em ambiente diferente que o descaracteriza partilhar o mesmo espaço físico com pessoas estranhas do seu convívio familiar e também pela preocupação, em relação a sua evolução do tratamento.(9)

Ainda, Bastos, ${ }^{\left({ }^{9}\right)}$ coloca que a intervenção cirúrgica caracteriza- se como traumática para a pessoa, por isso exige preparo e cuidado. A enfermagem tem o papel muito importante para garantir menores possibilidades de complicações, compreender tudo o que envolve o cuidado específico e singularizado. Saber ouvi-lo neste momento é crucial, aproxima o paciente do profissional, é neste momento que pode ser feito um bom acolhimento ao paciente por toda a equipe multiprofissional.

$\mathrm{Na}$ adesão ao checklist, precisa ser aprimorado todo o processo de trabalho dentro de uma instituição, onde, as diferenças sugerem, nas distintas estratégias de implantação utilizadas em cada instituição.

Esse instrumento, não vem substituir ou sistematizar a assistência de enfermagem e sim criar protocolos e a padronização para cada procedimento cirúrgico. Não esquecendo que essa equipe, também precisa ser capacitada e que receba educação permanente, para que todos da equipe trabalhe de forma padronizada para garantir segurança ao paciente.

Neste sentido, a importância do gerente de enfermagem para a organização e a Sistematização da Assistência e Enfermagem, afim de se ter uma excelência da qualidade e que o checklist vem para garantir a segurança na avaliação de enfermagem, na coleta de dados e exame físico, fazendo parte do processo de enfermagem e auxiliando no planejamento dos cuidados. Assim, torna-se necessária a avaliação dos fatores de risco de cada paciente no pré-operatório e a consideração desses fatores para elaboração do checklist. ${ }^{(12)}$ 


\section{Tema 2 - O papel da equipe de enfermagem frente ao cuidado no período pré-operatório}

Quando questionado ao profissional a sua atenção frente ao cuidado no pré-operatório, 16 profissionais, colocam sobre o cuidado com a segurança do paciente, sendo que, E1; E4, E19; E16; E5 enfatizam que "o papel é de manter o máximo de segurança ao paciente internado sobre os cuidados de enfermagem". Conforme um dos relatos: E19 - "Cuidado com estado emocional do paciente, acalmar deixar o paciente tranquilo e explicar como deve ser o procedimento que deve ser feito."

E outros 13, dizem que prestam conforto e cuidado humanizado e que "o conforto cuidado humanizado diminui riscos cirúrgicos para recuperação do paciente". Como podemos ver: E17; E15; E9; E15 - "Ter paciência saber entender cada situação, cada cirurgia acalmar os familiares e orientar o procedimento". E16 - "O enfermeiro deve estar à frente formulando o plano de cuidado, considerando os problemas de enfermagem que necessitam de intervenções para diminuir o risco cirúrgico." E17 "Simpatia empatia, deixa - ló confortável e seguro."

Já 08 profissionais relataram que o cuidado de enfermagem que realizam são os procedimentos técnicos necessários a cada paciente, a partir das consulta de enfermagem essa realizada na visita nos quartos para conhecer os paciente e necessidade de cada um, mesmo que possa acontecer fazer o mesmo procedimento mais são pessoas diferentes, à coleta de informações vão garantir a segurança do paciente, como podemos ver a seguir: E34; E18; E10 - "Muita responsabilidade." E7 - "O papel do técnico de enfermagem é proporcionar ao paciente, as melhores condições possíveis para que tudo ocorra dentro das normas, do que seria o ideal para uma assistência a esse paciente." E13 - "Verificação de todos os sinais vitais de forma correta passando no sistema, coletando todas as informações."

A visão dos profissionais em relação ao papel da equipe de enfermagem mostrou que tem que ter responsabilidade e trabalho técnico, mas demonstraram que não seguem um protocolo ou uma rotina, como também, não souberam responder sobre esta rotina.

Na pesquisa pode ser identificado que o hospital do estudo tem rotina para o preparo do paciente, mas que os trabalhadores não seguem esses cuidados, desta forma, podendo ser colocado em questão a segurança do paciente. 
A segurança cirúrgica consiste numa "sequência de etapas necessárias na assistência, não apenas pelo cirurgião, mas pela equipe de profissionais de assistência à saúde, trabalhando juntos em um sistema de saúde que os apoie para benefício do paciente". A sequência de etapas para a cirurgia segura inclui: "Prevenção de infecção no sítio cirúrgico; Anestesia segura; Equipes cirúrgicas eficientes; Mensuração da assistência segura".(2)

Desta forma, todas as pessoas que possam precisar de uma intervenção cirúrgica, precisam garantir um preparo e cuidado, desde o momento em que esse sujeito chega à instituição, onde, toda a equipe de enfermagem tem um papel primordial neste momento. ${ }^{(9)}$

Alguns dos profissionais não responderam (05).

\section{Tema 3 - Rotina ou checklist do serviço quanto o cuidado no período pré- operatório}

Se o serviço viabiliza uma rotina ou checklist de cuidado pré-operatório, 10 funcionários disseram que realiza, "mas que não tem por escrito e sim uma rotina apenas". Uns relataram que está sendo implantado, mas que não foi visto, e sim só um protocolo para as cirurgias cardíacas.

Considerando que as respostas são diversas quanto a ter uma rotina ou checklist, acredita-se que não sabem sobre este, pois junto as respostas apareceu justificativa de que a "rotatividade de funcionário é muito grande", e na "maioria das vezes funcionário novos não seguem rotina do setor no cuidado e preparo do paciente para cirurgia." Outros colocam que realmente não tem um checklist onde possam seguir para garantir a uma sequência do que deveria ser feito para cuidado e que poderia ser um bom instrumento para a segurança do paciente.

Como muitos dos profissionais tem pouco tempo de serviço neste setor, o que poderia ser realizado é a educação continuada para estes profissionais no que tange ao cuidado e segurança do paciente antes dos procedimentos cirúrgicos, cuidados esses necessários para evitar eventos adversos. Descrevemos algumas das falas: E18; E40; E39; E23; E22; E20 - "Não possui cheklist fora da sala cirúrgica. Uma boa opção seria a criação de um escrito para evitar erros". E42 - "O fluxo é muito intenso e na grande maioria das vezes não é feito da forma certa". 
Outros colocam que não tem o checklist no preparo dos pacientes para cirurgias, mas que tem uma rotina para cirurgias cardíacas, que o cirurgião descreve no prontuário do paciente. Relatam que "está sendo implantado, mas que não foi visto ainda." E12 - "Normalmente já tem uma rotina prescrita pelo médico assistente. Cheklist somente na cirurgia cardíaca, pois já tem uma rotina estabelecida."

Vários funcionários disseram que não tem (23), outros 9 não responderam.

A avaliação do uso do checklist em oito instituições-piloto no mundo mostrou que sua aplicação praticamente dobrou as chances dos usuários receberem o tratamento cirúrgico com padrões de cuidado adequados. Não se sabe o mecanismo responsável por esse evento, mas acredita-se que se deva a mudanças na rotina, no comportamento da equipe, de cada membro individual e coletivamente, e na comunicação interpessoal. ${ }^{(13)}$

Em 2008, o Ministério da Saúde do Brasil aderiu à campanha Cirurgias Seguras Salvam Vidas. ${ }^{(14)}$

A sensibilização para segurança do paciente deve ser pratica constante no serviço. Do gestor ao higienizador todos tem que ter a mesma postura para segurança do paciente, elevando e melhorando qualidade do serviço.

Assim, o Ministério da Saúde, institui ações para segurança do paciente nos serviços de saúde e sugere uma lista de verificação ou Checklist pela equipe de profissionais por especialistas, para ajudar as equipes cirúrgicas na redução de erros e danos ao paciente, e tem como um dos seus objetivos específicos produzir, sistematizar e difundir conhecimentos sobre segurança do paciente. E visando o alcance desse objetivo publicou por intermédio da Portaria Ministerial $\mathrm{n} \cong 1.377$, de 9 de julho de 2013 , os três primeiros protocolos que tratam das temáticas 'Cirurgia Segura', 'Prática de Higiene das mãos' e 'Úlcera por pressão'. Construídos a partir de consenso técnico-científico e considerando as sugestões recebidas por meio de consulta pública, visam contribuir para a qualidade e segurança do paciente. ${ }^{(15)}$

Portanto, os profissionais de enfermagem, devem ter muita atenção frente ao cuidado no pré-operatório, e para isso podem se calçar de um 'checklist' que pode ser utilizado nos diversos tipos de cirurgia, para realizar a mensuração dos vários itens presentes no instrumento e de referencial pela organização e funcionários, devido ao registro de cuidados com o paciente. ${ }^{(16)}$ 


\section{Tema 4 - Acompanhamento e orientação à família do paciente.}

No acompanhamento e orientações à família do paciente ou acompanhante, 28 profissionais relataram a pratica de dar informações dos procedimentos e cuidados que devem realizar antes da cirurgia, e que essas informações são repassadas aos familiares.

No deslocamento do paciente ao Centro Cirúrgico (CC), a família vai junto com paciente e o técnico de enfermagem. E39, E37, E23, E14, "Sim eles são encaminhado junto ao paciente ao CC antes da entrada para cirurgia e logo então encaminhado a sala de espera para posterior informação em horários." E39 - "Sim maiores informações são fornecidas pelos profissionais do CC.” E33 - "Quase sempre os médicos orientam a família."

Dos pesquisados, 08 deles responderam que às vezes é realizado 0 acompanhamento ao paciente e orientações à família. E6- fala que "quando solicitado pelo médico, o técnico vai passar informações para o paciente". Enquanto que 5 colocaram que "se for necessário dão orientação para familiares." Os outros 3 não responderam.

Nesta etapa do cuidado o paciente fica vulnerável, e o papel da equipe multiprofissional é orientar, cuidar e dar segurança ao paciente para que ele e seus familiares enfrentem melhor essa etapa.

O papel da equipe de enfermagem é orientar os pacientes e também os familiares no que diz respeito à cirurgia e todo o cuidado que vai precisar no preparo antes da cirurgia e após a cirurgia e para casa, cuidados esses necessários para sua segurança.

A hospitalização de um membro da família é um evento que gera estresse. Ansiedade da família pode ocorrer devido à incerteza sobre o prognóstico, à falta de privacidade e de individualidade. Ser familiar ou acompanhante é uma tarefa difícil, visto que as pessoas apresentam expectativas, ansiedades, preocupações, ambiente desconhecido e estressante. Muitos profissionais não percebem que esta família, também precisa de cuidado e apoio psicológico o e espiritual. (17) 


\section{Tema 5 - Outros cuidados realizados pela equipe de enfermagem}

Tanto a enfermagem, quanto a equipe multiprofissional precisa reorganizar o processo de trabalho, para dar resposta para o cuidado com paciente. Aqui, 19 dos profissionais não responderam quais outros cuidados a equipe de enfermagem realiza.

Os cuidados podem ser respondidos pela equipe de enfermagem desde 0 momento que o paciente entra no hospital, pois cuidados que o paciente não conhece pode gerar insegurança, medo, estresse, entre outros fatores. Aqui vem a importância de que todos os cuidados sejam realizados e anotados no prontuário do paciente.

Assim, destacamos a importância de ser realizada a visita ao paciente em seu leito, para conhecer a singularidade de cada um e realizar consulta de enfermagem, desta forma, proporcionando aos pacientes uma escuta qualificada e poder planejar o cuidado com eficiência e qualidade, resultando em pré-operatório eficaz e que ajude o paciente a lidar com os eventos adversos durante a cirurgia, reduzindo a permanência da internação hospitalar e elevando a satisfação com serviço prestado, minimizando as complicações cirúrgicas. ${ }^{(9)}$

\section{Tema 6 - Respostas colocadas pela equipe de enfermagem, quando o paciente chega no Centro Cirúrgico}

Destes, 33 profissionais participantes não responderam. E 05 profissionais responderam que deveria ter uma pessoa para receber o paciente no Centro Cirúrgico. É difícil o procedimento cirúrgico para paciente, por muitas vezes, faltar informação de como vai ser, quais as etapas antes e depois do ato cirúrgico. E10 "Uma Pessoa recebendo o paciente na porta do setor, seria interessante. Fazendo as rotinas necessárias deste paciente." E36, E13, E9, E6 - “Todo paciente ansioso, esse o risco de ir para cirurgia, mal preparado psicologicamente por isso tem que ter uma boa noite de sono."

Quando o paciente entra no centro cirúrgico, tudo é novo, acaba observando na entrada todo o movimento que acontece lá dentro e se deparando com uma tecnologia que para ele é desconhecido, mas que faz parte da segurança em 
prol do restabelecimento da saúde do paciente e visa solucionar e promover melhorias específicas em áreas de maior risco na assistência à saúde.

Outros responderam que falta trabalhar de forma holística, e que cada especialidade deve ter um protocolo. Um deles respondeu que falta conhecimento para o funcionário e informações ao paciente. E1 - "Venho dizer que temos que trabalhar muito, no pré e no pós-operatório. A enfermagem faz o que pode, mas na maioria dos casos o paciente não recebe informações necessárias de seu médico. Somos uma equipe multiprofissional, porém, temos que apreender usar os recursos necessários para o paciente, evitando conflitos e situações que podem prejudicar a saúde do paciente." E5 - "O preparo pré-operatório deveria ser protocolado para cada especialidade." E29 - "Essas perguntas, nos faz ver o nosso conhecimento, e perceber." E23 - "Quando o paciente está com dor, hipotensão, hipertenso, hipoglicemia, geralmente é medicada.".

O pré, trans e o pós-operatória, são fases todas muito importantes, mas a fase pré-operatória por considerá-la do período em que o paciente se encontra mais vulnerável em suas necessidades, tanto fisiológicas quanto psicológicas, tornando-se mais propenso a um desequilíbrio. Mas é na fase de pré-operatório imediato que se exige dos profissionais o preparo técnico científico, "desta forma, contribuindo com melhor atenção ao paciente nas demais fases do processo cirúrgico. Assim, exige-se não apenas o preparo técnico e teórico desses profissionais, mas também o humanístico'.(18)

Assim, o enfermeiro tem como uma de suas funções o planejamento e implementação de intervenções de enfermagem que minimizam ou possibilitam a prevenção de complicações decorrentes do procedimento anestésico e cirúrgico, visando a segurança, e conforto do paciente durante ato cirúrgico. Também a estrutura organizacional viável ao desenvolvimento do processo de trabalho dos profissionais e que tenham o conhecimento e o compromisso na reconstrução de práticas destinadas à qualidade da assistência, uma vez que toda transformação exige mobilização. ${ }^{(19)}$

A proposta de "Checklist" vem para contribuir e oportunizar maior segurança ao paciente em relação ao seu procedimento cirúrgico quanto à eficácia das atividades realizadas pela equipe, sendo assim é um instrumento que vai minimizar a ocorrência de intervenções erradas em instituições de saúde. Uma 
comunicação eficaz na equipe multidisciplinar, a marcação correta do local pelo cirurgião com o envolvimento do paciente, a revisão adequada do prontuário e dos equipamentos necessários ao procedimento cirúrgico, ajudam a instituição de saúde a determinar como este processo pode ser documentado de maneira resumida e objetiva através de um "checklist".

Assim, apresentamos a proposta elaborada do instrumento ou "checklist" a ser aplicado aos pacientes no seu pré-operatório. 
Quadro 1 - Proposta de Instrumento de Checklist em pré-operatório

\section{PROCEDIMENTOS PARA SEGURANÇA DO PACIENTE NO PRÉ- OPERATÓRIO - CHECKLIST}

\section{I) IDENTIFICAÇÃO:}

Nome:

Registro:

Enfermaria: Quarto/Leito: Idade:

Sexo: ( ) F; ( ) M

Data de Admissão: $1 \quad 1 \quad$ Data Cirurgia:

Cirurgia

Prevista/Local:

Lado: ( ) Dir.; ( ) Esq.; ( ) Bilateral; （ ) NSA (Não Se Aplica).

II) ANTES DO ENCAMINHAMENTO PARA O CENTRO CIRÚRGICO (PRÉOPERATÓRIO IMEDIATO: 24 HORAS ANTES DA CIRURGIA).

1. Paciente está com pulseira de identificação: ( ) SIM ; ( ) NÃO. Obs.

2. Paciente tem liberação anestésica: Termo consentimento assinado pelo paciente. () SIM; () NÃO. Obs.

3. Paciente tem alergia: ( ) SIM ; $($ NÃO. Especifique

4. Paciente está em jejum: ( ) SIM ; $($ ) NÃO. Obs.

5. Realizado preparo pré-operatório prescrito ou de rotina do serviço para cirurgia proposta (conforme P.O.P.): $\quad$ ( ) SIM ; ( ) NÃO; ( ) NSA.

Qual: ( ) Tricotomia; ( ) lavagem intestinal; ( ) higiene corporal e oral; ( ) banho com sabão antisséptico; ( ) Outros (especifique)

6. Foi retirada roupa íntima, acessórios, próteses e guardados pertences pessoais: ( ) SIM ; ( ) NÃO. ( ) Prótese Dentária; ( ) Prótese Ocular; ( ) Outros: 


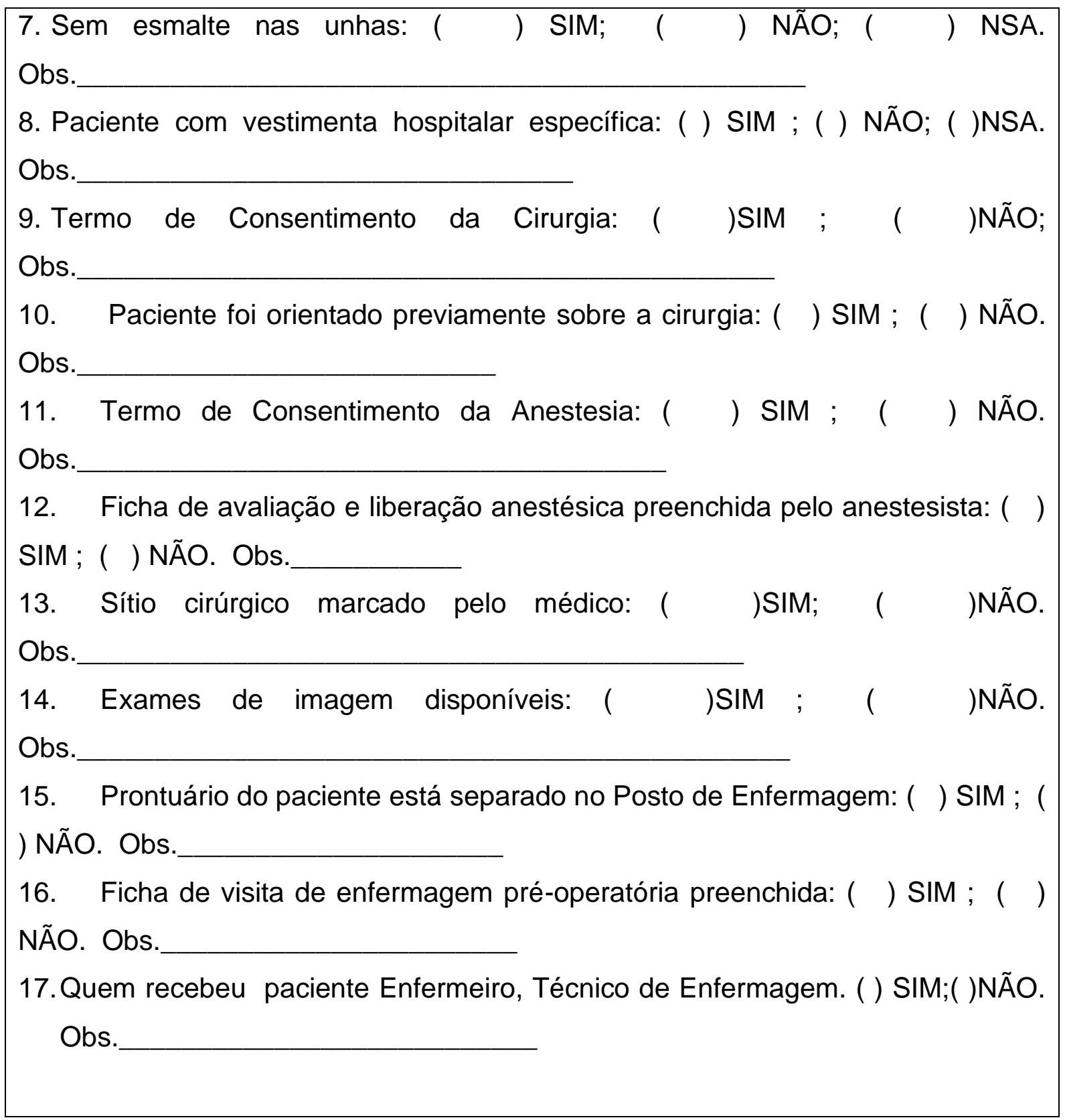

Fonte: da pesquisa, 2016

\section{CONSIDERAÇÕES FINAIS}

Pode-se ver que ainda existe deficiência no que se refere ao preparo físico e psicológico do paciente em pré-operatório para as cirurgias.

A internação para realizar uma cirurgia, na maioria das vezes é visto pelo paciente como um procedimento que exige dele um cuidado precoce que ele não compreende, muitas vezes para ele pode ser agressivo e invasivo. Assim, desde a 
internação ou a entrada na instituição, já deve acontecer o acolhimento e o preparo do paciente no período pré-operatório.

O objetivo de caracterizar os cuidados de enfermagem prestados a pacientes em período pré-operatório mediato e imediato e propor um "checklist" para a Clínica Cirúrgica, como forma de garantir a segurança do paciente antes do procedimento cirúrgico, foram alcançados e visto que a equipe de enfermagem precisa de "checklist", para poder melhorar a qualidade da assistência prestada e garantir segurança para o paciente no período pré-operatório.

Conhecer como ocorre o preparo do paciente antes do procedimento cirúrgico para garantir a cirurgia segura foi de grande valia para elaborar um "checklist". Esse instrumento não vem substituir ou sistematizar a assistência de enfermagem, que inclui os planos de cuidados e sua implementação, e sim criar uma padronização de pré-operatório para os procedimentos cirúrgicos ou formas de se desenvolver o cuidado, para alcançar resultados positivos e garantir a segurança aos pacientes, reduzindo os danos.

Enquanto que o "checklist" vem para garantir a segurança do paciente e assegurar que a instituição desenvolva suas atividades, eliminando erros possíveis durante um tratamento cirúrgico, a partir de práticas seguras baseadas em evidencias descritas, também promovendo condições para uma comunicação eficaz entre os membros da equipe multidisciplinar.

O planejamento é um instrumento e processo contínuo, que visa possibilitar uma postura ativa por parte da equipe de enfermagem para acontecer participação de todos e compreender que é necessário o comprometimento de toda equipe para o cuidado com o outro. A segurança é um indicador de qualidade, mas para que possa acontecer precisa que todos participem.

Portanto, com este estudo foi elaborado uma proposta de "checklist" para deixar na instituição pesquisada como proposta para capacitação da equipe de enfermagem e implementação deste instrumento. Proporcionar segurança aos pacientes em pré-operatório, aprimorará a comunicação eficaz na equipe de enfermagem e multidisciplinar, com o envolvimento do paciente, revisão adequada do prontuário e dos equipamentos necessários ao procedimento cirúrgico, desta forma, ajudando a instituição de saúde a determinar como este processo pode ser documentado de maneira resumida e objetiva através deste "checklist". 


\section{REFERÊNCIAS}

1 - Grigoleto, AR et al. Segurança do cliente e as ações frente ao procedimento cirúrgico. Rev Elet de Enferm. V. 13, n.2, p.347-54, abr/jun. 2011. Disponível em: http://www.fen.ufg.br/revista/v13/n2/v13n2a22.htm. Acesso em: 10 nov. 2015.

2 - Organização Mundial da Saúde. OMS. Segundo desafio global para a segurança do paciente: Cirurgias seguras salvam vidas. Rio de janeiro, 2009. Disponível em: http://proqualis.net/cirurgia/?id=000000483. Acesso em: 03 jun. 2015.

3 - Ferraz, EM. A cirurgia segura: Uma exigência do século XXI. Rev. Col. Bras. Cir. Rio de Janeiro, 2009, v. 39, n. 4, p. 281-282.

4 - Brasil. Agência Nacional de Vigilância Sanitária. Boletim Informativo- Segurança do paciente e qualidade em serviços de saúde. 2011. Disponível em: http://www.anvisa.gov.br/hotsite/notivisa/apresenta.htm. Acesso em: 04 jun. 2015.

5 - Nettina, SM. Prática de enfermagem. 6ª ed. RJ: Guanabara-Koogan, 2007.

6 - Christoforo, BEB; Carvalho, DS. Cuidados de enfermagem realizados ao paciente cirúrgico no período pré-operatório. Rev. esc. enferm. USP [online]. 2009, vol.43, n.1, pp.14-22. ISSN 1980-220X. http://dx.doi.org/10. 1590/S0080-62342009000100002.

7 - COREN, SP. Conselho Regional de São Paulo. 10 Passos para a segurança do paciente. São Paulo, 2010.

8 - Mafetoni, RR et al. Comunicação enfermeiro-paciente no pré-operatório: revisão integrativa. Rev Rene. V.12, n.4, p.859-865, Fortaleza, 2011.

9 - Bastos, $A Q$ et al. Reflexões sobre cuidados de enfermagem no pré e pósoperatório: uma revisão integrativa da literatura. Ciên e Cuidado a Saúde. Maringá, v. 12, n. 2, p. 382-390, abr.jun. 2013. 
10 - Maciel, MED; Oliveira, FN. Qualidade de vida do profissional técnico de enfermagem: a realidade de um hospital filantrópico em Dourados-MS. Rev. Psicol. Saúde, Campo Grande, v. 6, n. 1, p. 83-89, jun. 2014. Disponível em http://pepsic.bvsalud.org/scielo. Php?Script=sci_arttext\&pid=S2177093X2014000100011\&lng=pt\&nrm=iso. Acessos em 27 jun. 2016.

11 - Santana, VS et al. Qualidade de vida dos profissionais de saúde em ambiente hospitalar. Rev Pesq em Fisioterapia.; v.4(1):35-46, abr 2014.

12 - Soares, Ml et al. Sistematização da assistência de enfermagem: facilidades e desafios do enfermeiro na gerência da assistência. Esc. Anna Nery. Rio de Janeiro, v. 19, n. 1, p. 47-53, mar. 2015. Disponível em: http://www.scielo.br/scielo. php?Script=sci_arttext\&pid=S1414-81452015000100047\&lng=en\&nrm=iso. Acesso em: 09 jun. 2016. http://dx.doi.org/10. 5935/1414-8145.20150007

13 - Pancieri, AP et al. Checklist de cirurgia segura: análise da segurança e comunicação das equipes de um hospital escola. Revista Gaúcha de Enfermagem. São Paulo, p.71-78, 2014.

14 - Motta Filho, GR et al. The WHO Surgical Safety Checklist: knowledge and use by Brazilian orthopedists. Rev. bras. Ortop. [online]. 2013, vol. 48, n.6, pp.554-562. ISSN 0102-3616. http://dx.doi.org/10.1016/j.rboe.2013.12.010.

15 - Brasil. ANVS. Boletim Informativo- Segurança do paciente e qualidade em serviços de saúde. 2011.2 Disponível em: http://www.anvisa.gov.br/hotsite/notivisa/apresenta.htm. Acesso em: 04 jun. 2015.

16 - JCAHO - Joint commission on acreditation of healthcare organizations - Joint Commission International. Padrões de acreditação da Joint Commission International para hospitais. 4. Ed. Salvador, 2010. Disponível em: http://www.Jointcomissioninternational.org/hospital/fourth_edition_hospital_manual_ portuguese_trranslation.pdf. Acesso em: 03 jun. 2015. 
17 - Peres, GM; Lopes, AMP. Acompanhamento de pacientes internados e processos de humanização em hospitais gerais. Psicol. Hosp. São Paulo, v. 10, n. 1, p. 17-41, jan. 2012. Disponível em: http://pepsic.bvsalud.org/scielo. Php?Script=sci_arttext\&pid=S1677-74092012000100003\&lng=pt\&nrm=iso. Acesso em: 09 jun. 2016.

18 - Costa, VASF et al.O pré-operatório e a ansiedade do paciente: a aliança entre o enfermeiro e o psicólogo. Rev. SBPH. Rio de Janeiro, v. 13, n. 2, p. 282-298, dez. 2010. Disponível em http://pepsic.bvsalud.org/scielo. Php?Script=sci_arttext\&pid=S1516-08582010000200010\&lng=pt\&nrm=iso. Acesso em: 09 jun. 2016.

19 - Lopes, CMM; Galvão, CM. Surgical Positioning: Evidence for Nursing Care. Rev. Latino-Am. Enfermagem [online]. vol.18, n.2, pp.287-294, 2010. Disponível em: http://dx.doi.org/10. 1590/S0104-11692010000200021. Acesso em 10 maio 2016. 